\title{
FAM3C activates HSF1 to suppress hepatic gluconeogenesis and attenuate hyperglycemia of type 1 diabetic mice
}

\author{
Zhenzhen Chen ${ }^{1,2, *}$, Junpei Wang ${ }^{2, *}$, Weili Yang ${ }^{2}$, Ji Chen ${ }^{2}$, Yuhong Meng ${ }^{2}$, Biaoqi \\ Feng ${ }^{2}$, Yujing $\mathrm{Chi}^{3}$, Bin Geng ${ }^{1}$, Yong Zhou ${ }^{4}$, Qinghua Cui ${ }^{2,5}$ and Jichun Yang ${ }^{2}$ \\ ${ }^{1}$ Hypertension Center, Fuwai Hospital, Chinese Academy of Medical Sciences and Peking Union Medical College, State Key \\ Laboratory of Cardiovascular Disease, National Center for Cardiovascular Diseases, Beijing 100037, China \\ ${ }^{2}$ Department of Physiology and Pathophysiology, School of Basic Medical Sciences, Key Laboratory of Molecular Cardiovascular \\ Science of The Ministry of Education Center for Non-Coding RNA Medicine, Peking University Health Science Center, Beijing \\ 100191, China \\ ${ }^{3}$ Institute of Clinical Molecular Biology \& Central Laboratory, Peking University People's Hospital, Beijing 100044, China \\ ${ }^{4}$ Beijing Institute of Heart, Lung and Blood Vessel Diseases, Beijing Anzhen Hospital, Capital Medical University, Beijing \\ 100029, China \\ ${ }^{5}$ Department of Biomedical Informatics, School of Basic Medical Sciences, Key Laboratory of Molecular Cardiovascular \\ Science of The Ministry of Education, Center for Non-Coding RNA Medicine, Peking University Health Science Center, Beijing \\ 100191, China \\ *These authors have contributed equally to this work \\ Correspondence to: Jichun Yang, email: yangj@bjmu.edu.cn \\ Keywords: FAM3C; hepatokine; HSF 1; type 1 diabetes; gluconeogenesis \\ Received: July 06, $2017 \quad$ Accepted: October 30, $2017 \quad$ Published: November 20, 2017 \\ Copyright: Chen et al. This is an open-access article distributed under the terms of the Creative Commons Attribution License 3.0 \\ (CC BY 3.0), which permits unrestricted use, distribution, and reproduction in any medium, provided the original author and source \\ are credited.
}

\section{ABSTRACT}

FAM3C, a member of FAM3 gene family, has been shown to improve insulin resistance and hyperglycemia in obese mice. This study further determined whether FAM3C functions as a hepatokine to suppress hepatic gluconeogenesis of type 1 diabetic mice. In STZ-induced type 1 diabetic mouse liver, the FAM3C-HSF1-CaM signaling axis was repressed. Hepatic FAM 3 C overexpression activated HSF1-CaM-Akt pathway to repress gluconeogenic gene expression and ameliorate hyperglycemia of type 1 diabetic mice. Moreover, hepatic HSF1 overexpression also activated CaM-Akt pathway to repress gluconeogenic gene expression and improve hyperglycemia of type 1 diabetic mice. Hepatic FAM3C and HSF1 overexpression had little effect on serum insulin levels in type 1 diabetic mice. In cultured hepatocytes, conditioned medium of Ad-FAM3C-infected cells induced Akt phosphorylation. Moreover, Akt activation and gluconeogenesis repression induced by FAM3C overexpression were reversed by the treatment with anti-FAM3C antibodies. Treatment with recombinant FAM3C protein induced Akt activation in a HSF1- and CaM-dependent manner in cultured hepatocytes. Furthermore, recombinant FAM $3 C$ protein repressed gluconeogenic gene expression and gluconeogenesis by inactivating FOXO1 in a HSF1-dependent manner in cultured hepatocytes. In conclusion, FAM3C is a new hepatokine that suppresses hepatic gluconeogenic gene expression and gluconeogenesis independent of insulin by activating HSF1-CaM-Akt pathway. 


\section{INTRODUCTION}

Diabetes has become a severe worldwide epidemic affecting more than 400 million people [1] Excessive hepatic gluconeogenesis due to insulin resistance or insulin deficiency plays crucial roles in the development of diabetes [2]. Type 1 diabetes is mainly due to auto-immune-mediated pancreatic beta cell destruction, resulting in the deficiency of circulating insulin [3]. FOXO1 is the key transcriptor regulating gluconeogenesis, and it is phosphorylated and inactivated by insulin-mediated Akt activation in physiological condition [4]. Overactivation of hepatic FOXO1 due to insulin deficiency or insulin resistance will trigger excessive gluconeogenesis and hyperglycemia. Insulin and insulin analogues are the main therapeutical agents for type 1 diabetes [5]. However, it had been revealed that drugs that suppress hepatic gluconeogenesis also help reduce blood glucose levels in type 1 diabetic patients. For example, adjunctive metformin therapy with insulin reduces $\mathrm{HbAlc}$ levels and daily insulin dosage $[6,7]$. Identifying new genes or pathways that activate Akt to repress hepatic FOXO1 activity independent of insulin will shed light on the treatment of both type 1 and type 2 diabetes.

Family with sequence similarity 3 (FAM3) gene family consists of four members designated as FAM3A, FAM3B, FAM3C and FAM3D, respectively [8]. In the past decade, FAM3B, also called pancreatic derived factor (PANDER), had been revealed to be a negative regulator of pancreatic beta cell function and liver insulin signaling [9-14]. FAM3A is a novel mitochondrial protein that plays important roles in suppressing hepatic gluconeogenesis and lipogenesis by activating the ATP-P2 receptor-Akt pathway [15-17]. FAM3A also protect against liver ischemia/reperfusion injury [18]. More recently, we had demonstrated that FAM3C expression is reduced in the livers of obese mice. Hepatic FAM3C overexpression improves insulin resistance, hyperglycemia and steatosis of obese mice [19]. Mechanistically, FAM3C activates transcriptor heat shock factor 1 (HSF1) to induce CALM1 gene expression and increase calmodulin $(\mathrm{CaM})$ protein level. An increase in CaM protein level finally suppresses glucose production by inducing Akt activation in hepatocytes [19]. Clearly, FAM3C represents a new target for treating type 2 diabetes. However, several issues still remain unaddressed in our previous study. One is that although FAM3C activates HSF1-CaM pathway to activate Akt independent of insulin in cultured hepatocytes, whether it can suppress hepatic gluconeogenesis to improve hyperglycemia of type 1 diabetic mice remains unknown. Generally, two FAM3C protein isoforms exist in hepatocytes [19], one is the full length form (FL, 26kD) and the other is the secretory form (SF, 22kD) [19, 20]. So, another important issue is that whether secretion is necessary for FAM3C's effects of activating Akt and suppressing gluconeogenesis in hepatocytes, particularly in the condition of FAM3C overexpression or upregulation. Addressing these issues is necessary for the establishment that FAM3C functions as a new hepatokine to suppress hepatic gluconeogenesis independent of insulin.

In the current study, we demonstrated that hepatic FAM3C or HSF 1 overexpression repressed gluconeogenic gene expression and attenuated hyperglycemia of type 1 diabetic mice. FAM3C secretion is necessary for its effects of activating Akt and repressing gluconeogenesis in hepatocytes. Treatment with recombinant FAM3C protein (rFAM3C) repressed gluconeogenic gene expression and gluconeogenesis by activating Akt to repress FOXO1 activity in a HSF1-CaM-dependent manner in hepatocytes. Overall, these findings revealed that FAM $3 \mathrm{C}$ is a new hepatokine that suppresses hepatic gluconeogenesis independent of insulin by activating HSF1-CaM-Akt pathway.

\section{RESULTS}

\section{FAM3C-HSF1-CaM pathway was repressed in the livers of type 1 diabetic mice}

To determine whether FAM3C-HSF1-CaM pathway is involved in increased hepatic glucose production under type 1 diabetic condition, their expression levels in STZ-treated diabetic mouse livers were analyzed. One month later, the fasting blood glucose levels of STZ-treated mice were markedly elevated when compared with control mice (Figure 1A). In type 1 diabetic mouse livers, the mRNA levels of FAM3C, HSF1 and CALM1 were reduced when compared with control mouse livers (Figure 1B). In support, the protein levels of both FAM3C isoforms, HSF 1 and CaM were also reduced in type 1 diabetic mouse livers when compared with normal mouse livers (Figure 1C-1D).

\section{Hepatic overexpression of FAM3C or HSF1 attenuated fasting hyperglycemia of type 1 diabetic mice}

To directly evaluate the effects of FAM3CHSF1-CaM pathway on hepatic gluconeogenesis and hyperglycemia of type 1 diabetic mice, FAM3C or HSF1 was overexpressed in STZ-treated mouse livers via tail vein injection of Ad-FAM3C or HSF1 plasmid. At 3 and 7 days post Ad-FAM3C injection, the fasting blood glucose levels were significantly reduced when compared with mice treated with Ad-GFP (Figure 2A). 
Particularly, injection of Ad-FAM3C almost reduced the fasting blood glucose levels of diabetic mice to that of normal mice post 7 days (Figure 2A). The serum insulin levels between Ad-GFP- and Ad-FAM3C-treated mice were not different (Figure 2B). The mRNA levels of insulin genes in Ad-GFP- and Ad-FAM3C-treated mouse pancreas were also not different (Figure 2C). Injection of Ad-FAM3C increased the mRNA levels of HSF1 and CALM1, while reduced that of PEPCK and G6Pase in diabetic mouse livers (Figure 2D). Injection of Ad-FAM3C increased both FAM3C protein isoforms in diabetic mouse livers (Figure 2E-2F). FAM3C overexpression increased the protein levels of HSF1, $\mathrm{CaM}$ and phosphorylated Akt (pAkt) with the reduced PEPCK and G6Pase protein levels in type 1 diabetic mouse livers (Figure 2E-2F).

At 3 days post HSF1 plasmid injection, the fasting blood glucose levels were decreased when compared with diabetic mice treated with pGFP plasmid (Figure $3 \mathrm{~A})$. Injection of HSF 1 plasmid had little effect on serum insulin levels and insulin gene expression in pancreas

A

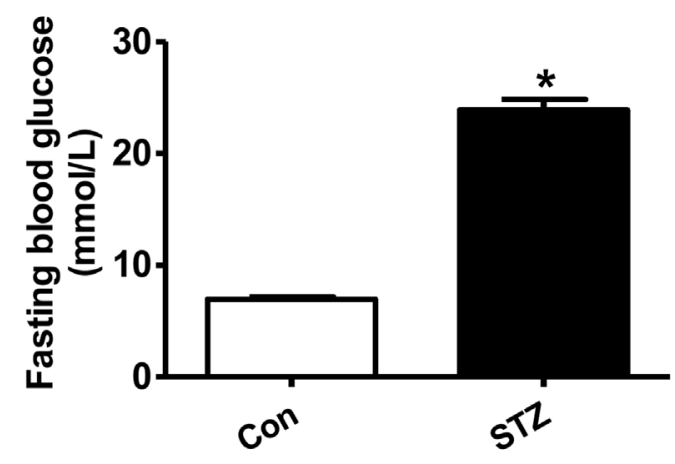

B

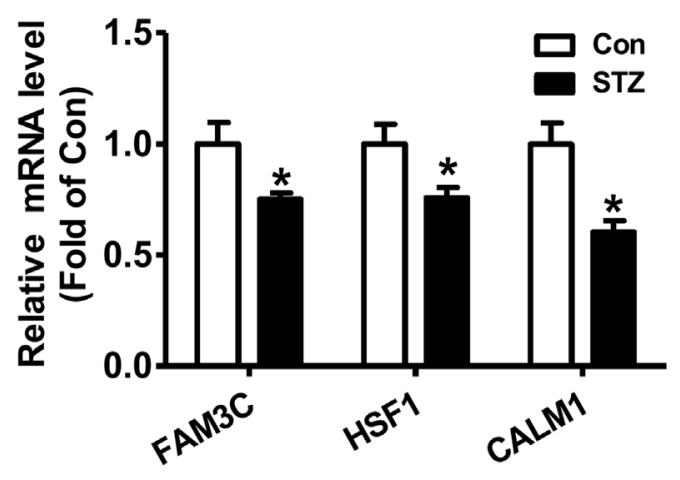

(Figure 3B-3C). Injection of HSF1 plasmid increased the mRNA levels of HSF1 and CALM1, while reduced that of PEPCK and G6Pase in type 1 diabetic mouse livers (Figure 3D). HSF1 overexpression increased the protein levels of CaM and pAkt but decreased that of PEPCK and G6Pase in diabetic mouse livers (Figure 3E$3 \mathrm{~F}$ ). Overall, the changes in hepatic Akt phosphorylation and gluconeogenic gene expression were consistent with the amelioration of hyperglycemia after FAM3C or HSF1 overexpression.

\section{FAM3C secretion is necessary for its effects on Akt activation and gluconeogenesis repression in cultured hepatocytes}

To determine whether or not secretion is necessary for FAM3C-induced Akt activation, HepG2 cells were treated with conditioned medium of Ad-GFP- or Ad-FAM3C-treated cells, respectively. As a result, conditioned medium of Ad-FAM3C-treated cells induced Akt activation in HepG2 cells (Figure 4A). In support,

C

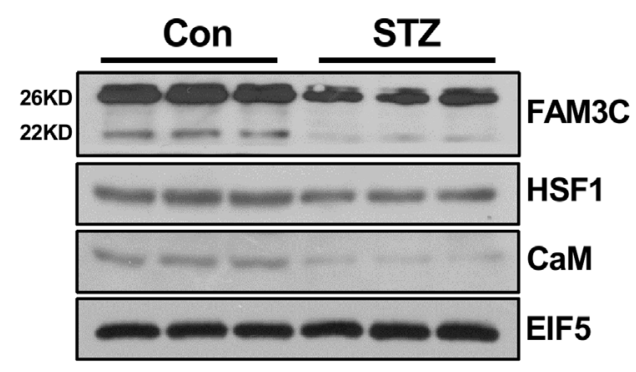

D

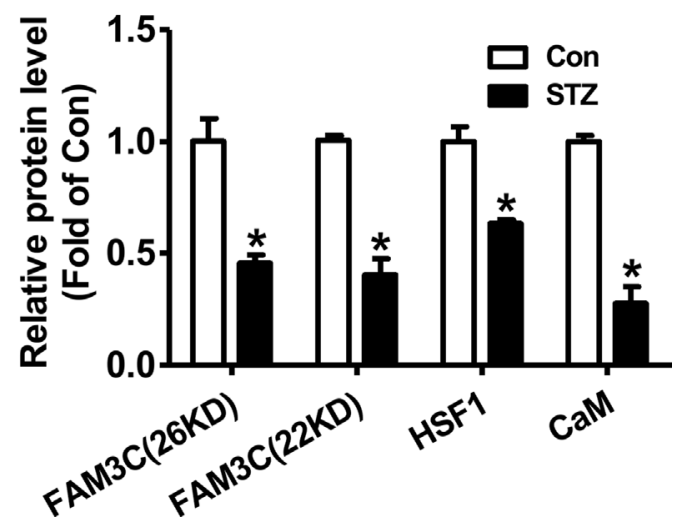

Figure 1: FAM3C-HSF1-CaM pathway was repressed in type 1 diabetic mouse livers. (A) Fasting blood glucose levels of mice were markedly elevated at 1 month post STZ injection. (B) The mRNA levels of FAM3C, HSF1 and CALM1 were reduced in diabetic mouse livers. (C-D) The protein levels of FAM3C, HSF1 and CaM were reduced in diabetic mouse livers. The representative gel images were shown in panel $\mathrm{C}$, and quantitative data in panel D. Con, control mice treated with saline; STZ, mice treated with $\mathrm{STZ}$. N=6-8, ${ }^{*} \mathrm{P}<0.05$ versus control mice. 
Akt activation induced by FAM3C overexpression was inhibited by treatment with anti-FAM3C antibodies in HepG2 cells (Figure 4B). Anti-FAM3C antibody treatment had little effect on FAM3C expression in the cells (Figure 4B). What's more, gluconeogenesis repression induced by FAM3C overexpression was also reversed by treatment with anti-FAM3C antibodies (Figure 4C). In support, conditioned medium of Ad-FAM3C-treated mouse hepatocytes also induced Akt phosphorylation in primary mouse hepatocytes (Figure 5A). Akt phosphorylation and gluconeogenesis suppression induced by FAM3C overexpression were also reversed by the treatment of anti-FAM3C antibodies in primary mouse hepatocytes (Figure 5B-5C).

\section{Recombinant FAM3C protein activated Akt pathway to repress gluconeogenesis in a HSF1- and CaM-dependent manner in hepatocytes}

To further confirm that secretion is necessary for FAM3C-induced activation of HSF1-CaM-Akt pathway, human HepG2 cells and mouse hepatocytes were treated with rFAM3C. As a result, rFAM3C dose-dependently induced Akt phosphorylation in HepG2 cells (Figure 6A). Importantly, Akt phosphorylation induced by rFAM3C was blocked by HSF1 inhibitor KRIBB11 and CaM inhibitor CPZ in HepG2 cells (Figure 6B). Moreover, rFAM3C similarly induced Akt phosphorylation in primary mouse hepatocytes (Figure 6C). Inhibition of HSF1 also abolished rFAM3C-induced Akt activation in primary mouse hepatocytes (Figure 6C).

Physiologically, activation of Akt suppresses gluconeogenic gene expression and gluconeogenesis by promoting the nuclear exclusion of FOXO1, a key transcriptor controlling the transcription of crucial gluconeogenic genes such as PEPCK [4]. Consistent with the Akt activation, rFAM3C treatment promoted the nuclear exclusion of FOXO1 in HepG2 cells (Figure 7A). In support of FOXO1 inactivation, rFAM3C significantly reduced the PEPCK protein expression in HepG2 cells (Figure 7B). Moreover, rFAM3C also repressed PEPCK expression in primary mouse
A
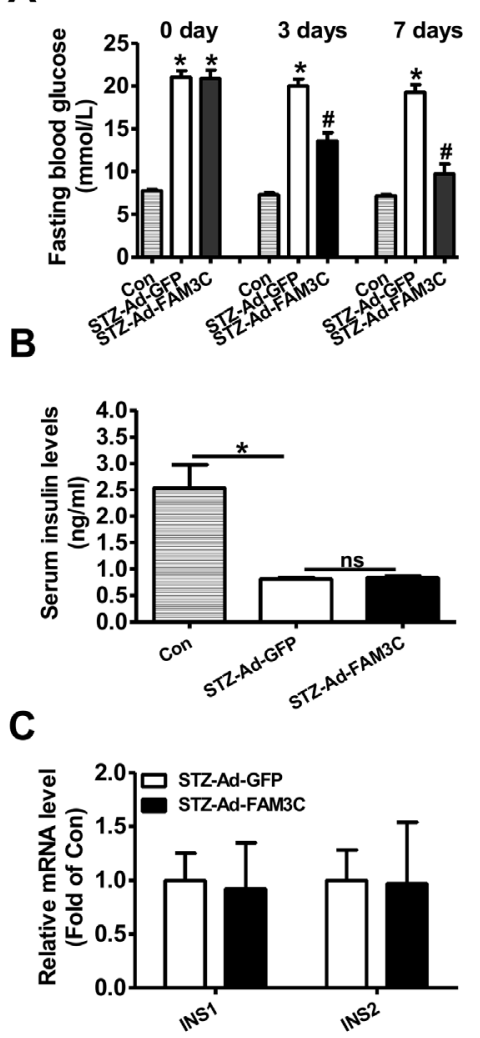

D

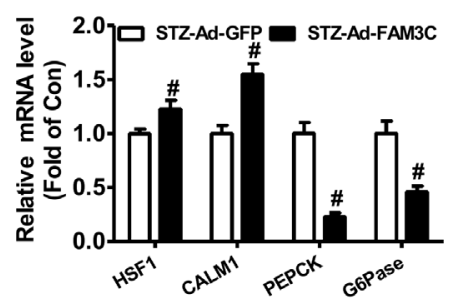

$\mathbf{E}$
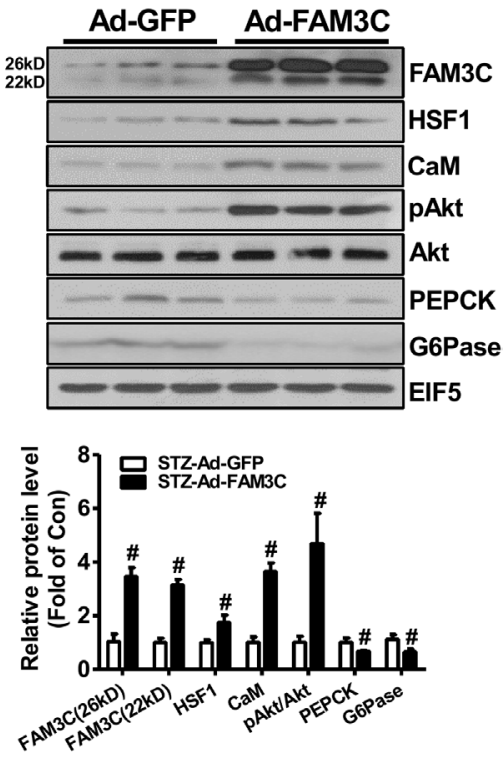

Figure 2: Adenoviral overexpression of FAM3C in type 1 diabetic mouse livers attenuated hyperglycemia. Mice were transduced with Ad-FAM3C or Ad-GFP as detailed in the experimental procedure. (A) Fasting blood glucose levels of type 1 diabetic mice before and after Ad-FAM3C injection. (B) Serum insulin levels after Ad-FAM3C injection. (C) Insulin mRNA expression levels in pancreas of mice after Ad-FAM3C injection. (D) FAM3C overexpression on the mRNA levels of metabolic genes in diabetic mouse livers. N=8-10, ${ }^{*} \mathrm{P}<0.05$ versus control mice, ${ }^{\#} \mathrm{P}<0.05$ versus STZ-Ad-GFP mice. (E-F) FAM3C overexpression on the protein levels of metabolic genes in diabetic mouse livers. Con, control mice; STZ-Ad-GFP, STZ-treated mice transduced with Ad-GFP; STZ-Ad-FAM3C, STZ-treated mice transduced with Ad-FAM3C. $\mathrm{N}=6-8,{ }^{\#} \mathrm{P}<0.05$ versus STZ-Ad-GFP mice. 
hepatocytes (Figure 7C). rFAM3C-triggered FOXO1 nuclear exclusion was further shown to be blocked by the inhibition of HSF1 using KRIBB11 in HepG2 cells (Figure 8A). Moreover, rFAM3C-induced repression on PEPCK expression was also reversed by inhibiting HSF1 (Figure 8B). In support of repression of FOXO1 activity and PEPCK expression, rFAM3C significantly repressed glucose production (Figure 8C). Importantly, rFAM3C-induced repression on gluconeogenesis was also reversed by inhibiting HSF1 in HepG2 cells (Figure 8C). Overall, rFAM3C induced Akt activation and repressed gluconeogenesis in hepatocytes via HSF1CaM pathway as FAM3C overexpression [19].

\section{DISCUSSION}

In the current study, we provided new evidences that FAM3C activated HSF1-CaM-Akt pathway to repress hepatic gluconeogenic gene expression and attenuate hyperglycemia of type 1 diabetic mice. These findings and the previous findings together revealed that FAM3C suppresses hepatic gluconeogenesis independent of insulin in vitro and in vivo. What's more, we provided additional evidences that secretion of FAM3C protein is important for its effects of inducing Akt phosphorylation and repressing gluconeogenesis in cultured human and mouse hepatocytes. Clearly, FAM3C is a new hepatokine. Our previous and current findings revealed hepatic FAM3C expression will regulate glucose metabolism by impacting on liver and other tissues as both an autocrine and an endocrine factor (Figure 9). So far, the FAM3C receptor still remains unknown. To identify the FAM3C receptor and determine its tissue distribution will be crucial for understanding FAM3C signaling transduction and its overall effects in glucose metabolism. This also suggested that circulating FAM3C could be a new biomarker for diagnosis and classification of diabetes, particularly in the era of precision medicine. Beyond
A

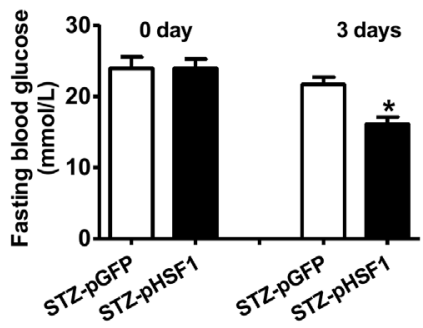

B

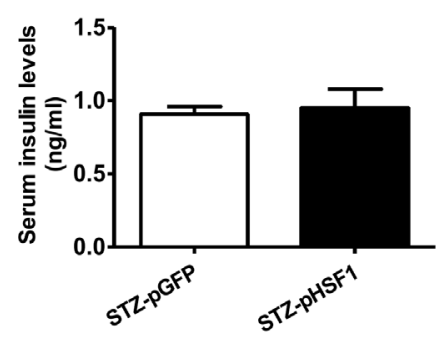

C

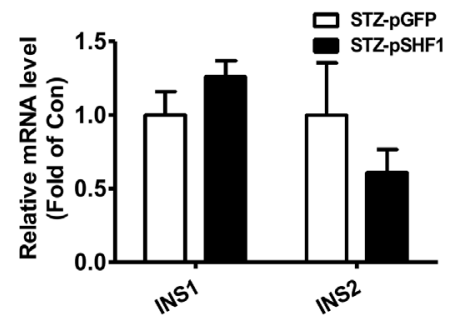

D

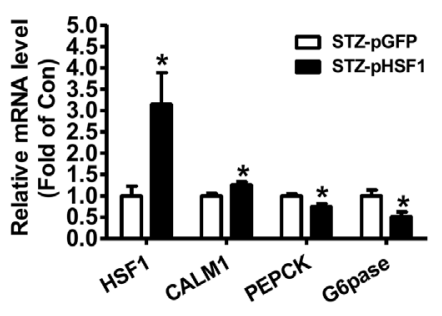

E
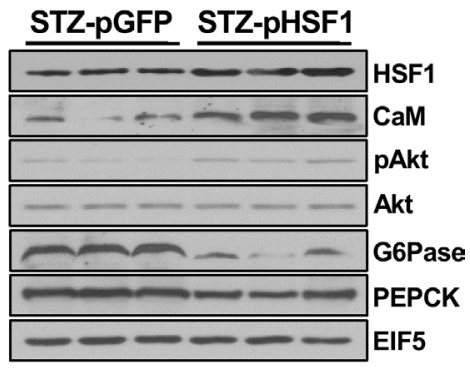

F

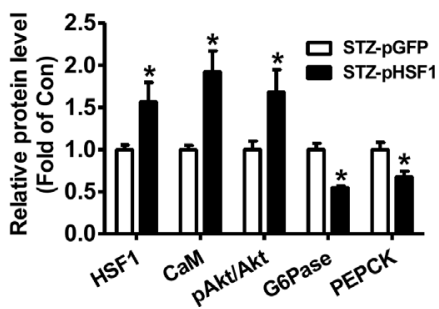

Figure 3: Plasmid overexpression of HSF1 in the livers improved hyperglycemia of type 1 diabetic mice. Diabetic mice were transfected with plasmid as described in the experimental procedure. (A) Fasting blood glucose levels of diabetic mice before and after plasmid transfection. (B) Serum insulin levels after plasmid transfection. (C) Insulin mRNA expression levels in pancreas of mice after plasmid transfection. (D) Injection of HSF1 plasmid on the mRNA levels of metabolic genes in diabetic mouse livers. $\mathrm{N}=8,{ }^{*} \mathrm{P}<0.05$ versus mice STZ-pGFP mice. (E-F) HSF1 overexpression on the protein levels of metabolic genes in diabetic mouse livers. STZ-pGFP, STZ-treated mice transfected with GFP plasmid; STZ-pHSF1, STZtreated mice transfected with HSF1 plasmid. N=6-8, versus STZ-pGFP group of mice. 
diabetes, dysregulated glucose and lipid metabolism are also highly associated with various cancers [22]. FAM3C expression is upregulated in several cancers, and circulating FAM3C level could also be a biomarker for autophagy and some cancers [23-25]. Anti-diabetic drug metformin exerts beneficial effects in various cancers in animals and humans [26]. Under conditions with dysregulated glucose and lipid metabolism, serum FAM3C protein level might be a novel biomarker for predicting the risk of diabetes or cancer. So far, there is no effective and sensitive method for detecting secreted FAM3C protein in the circulation of human and animals, and in cell culture medium. To develop high-sensitive FAM3C detection kit is necessary for establishing FAM3C as a new biomarker for both diabetes and cancer. PANDER (FAM3B) is processed by cleaving the signal peptide and then secreted from pancreatic alpha and beta cells, and secreted PANDER induces hepatic insulin resistance $[14,27,28]$. An elevation in circulating PANDER level is associated with insulin resistance and hyperglycemia in a Chinese population [29]. However, although liver-produced PANDER also plays important roles in regulating glucose and lipid metabolism, only one PANDER protein isoform (full length form, 27kD) can be detected in mouse livers and cultured hepatocytes with or without PANDER overexpression [9, 10, 13, 30], suggesting that PANDER may not be processed and released from hepatocytes.

Recently, it had been realized that cellular stress response plays important roles in the pathogenesis of type 2 diabetes $[31,32]$. When cells are exposed to stressors such as heat, radiation and oxidative stress, the expression of heat shock proteins (HSP) will be induced to protect cells against the damages triggered by these deleterious factors. These HSP are also called inducible HSP (iHSP) [31, 32]. The main functions of iHSP include assisting the folding of new or denatured proteins, preventing protein degradation and removing the nonfunctional proteins under stressed conditions [31-33]. As detailed in review [32], the expression levels of iHSP and their response to stressors are impaired in some important metabolic tissues including
A

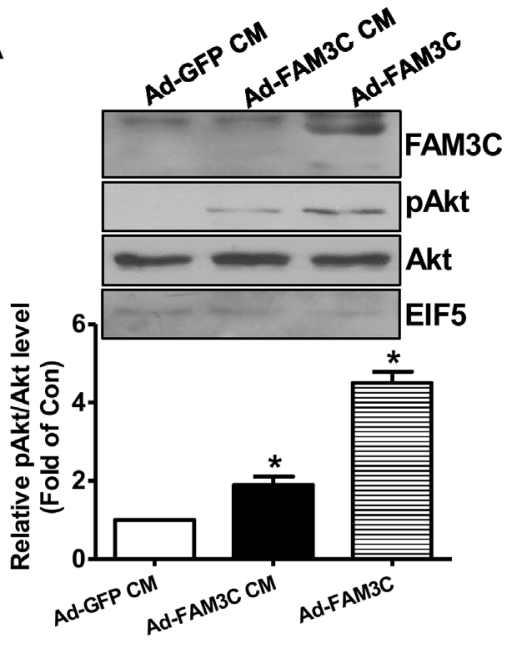

C

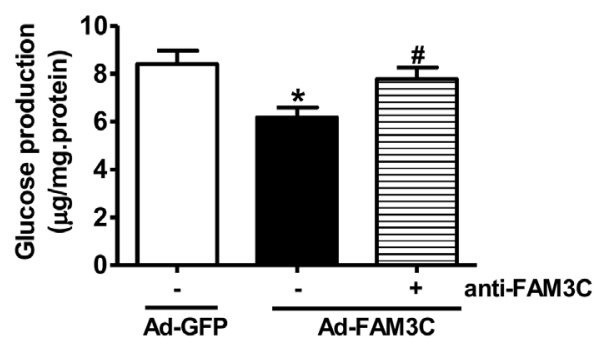

B Ad-GFP AdFAM3C

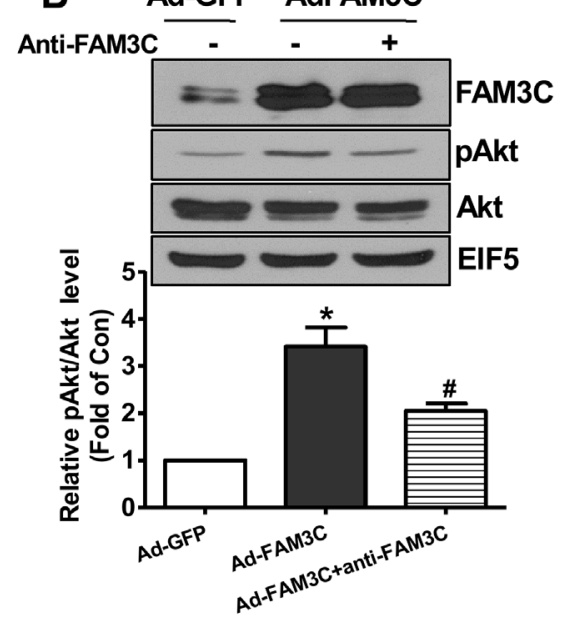

Figure 4: Secretion is important for FAM3C-induced Akt activation and gluconeogenesis repression in HepG2 cells. (A) Conditioned medium of Ad-FAM3C-infected cells induced Akt phosphorylation in HepG2 cells. HepG2 cells were treated with conditioned medium of Ad-GFP- or Ad-FAM3C-infected cells for 24 hours before Akt phosphorylation was assayed. Ad-FAM3C-infected cells was used as a positive control. $\mathrm{N}=3,{ }^{*} \mathrm{P}<0.05$ versus cells treated with conditioned medium of Ad-GFP-infected cells. (B) Treatment with anti-FAM3C antibodies inhibited Akt activation induced by FAM3C overexpression in HepG2 cells. (C) Treatment with anti-FAM3C antibodies reversed FAM3C-induced repression on gluconeogenesis in HepG2 cells. CM, conditioned medium. $\mathrm{N}=4-5$, ${ }^{*} \mathrm{P}<0.05$ versus Ad-GFP-infected cells, ${ }^{\#} \mathrm{P}<0.05$ versus Ad-FAM3C-infected cells. 

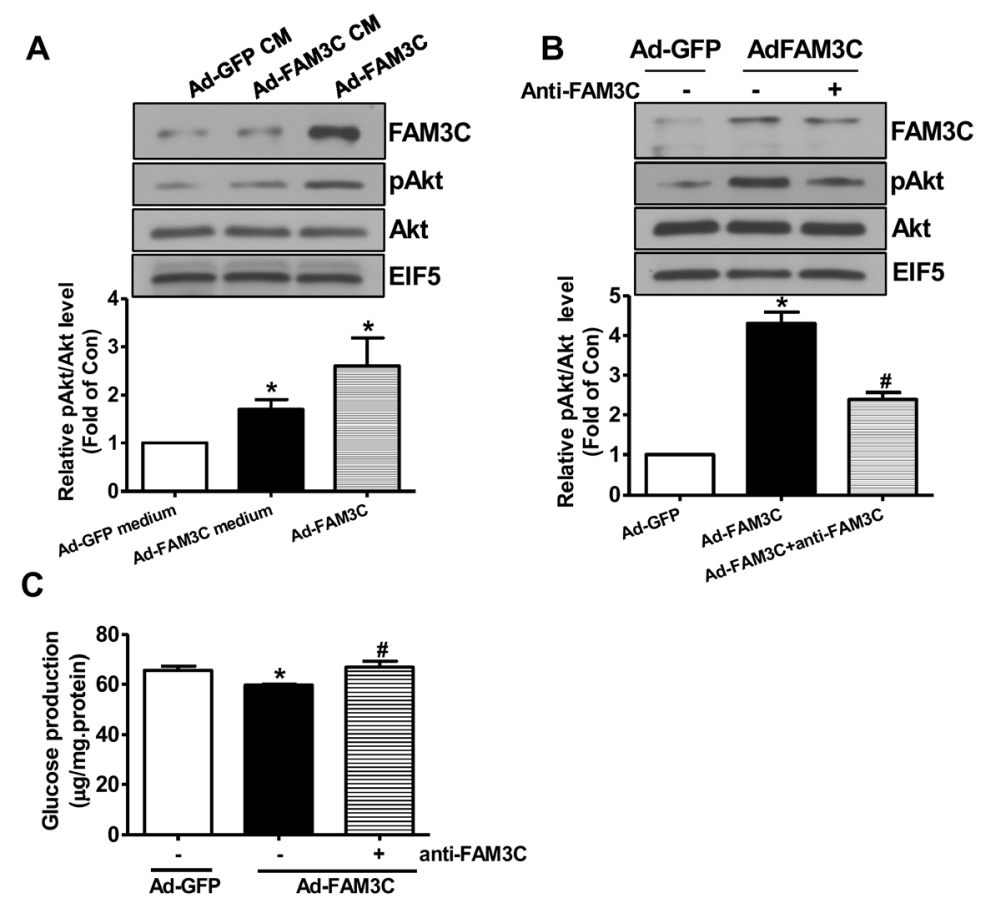

Figure 5: Secretion is imporant for FAM3C-induced Akt activation and gluconeogenesis repression in primary mouse hepatocytes. (A) Conditioned medium of Ad-FAM3C-infected cells induced Akt phosphorylation in primary mouse hepatocytes. Mouse hepatocytes were treated with conditioned medium of Ad-GFP- or Ad-FAM3C-infected hepatocytes for 24 hours before Akt phosphorylation was assayed. Ad-FAM3C-infected hepatocytes was used as a positive control. $\mathrm{N}=3,{ }^{*} \mathrm{P}<0.05$ versus cells treated with conditioned medium of Ad-GFP-infected cells. (B) Treatment with anti-FAM3C antibodies inhibited Akt activation induced by FAM3C overexpression in primary mouse hepatocytes. (C) Treatment with anti-FAM3C antibodies reversed FAM3C-induced repression on gluconeogenesis in primary mouse hepatocytes. CM, conditioned medium. $\mathrm{N}=4$, ${ }^{*} \mathrm{P}<0.05$ versus Ad-GFP-infected cells, ${ }^{\mathrm{P}} \mathrm{P}<0.05$ versus Ad-FAM3C-infected cells.

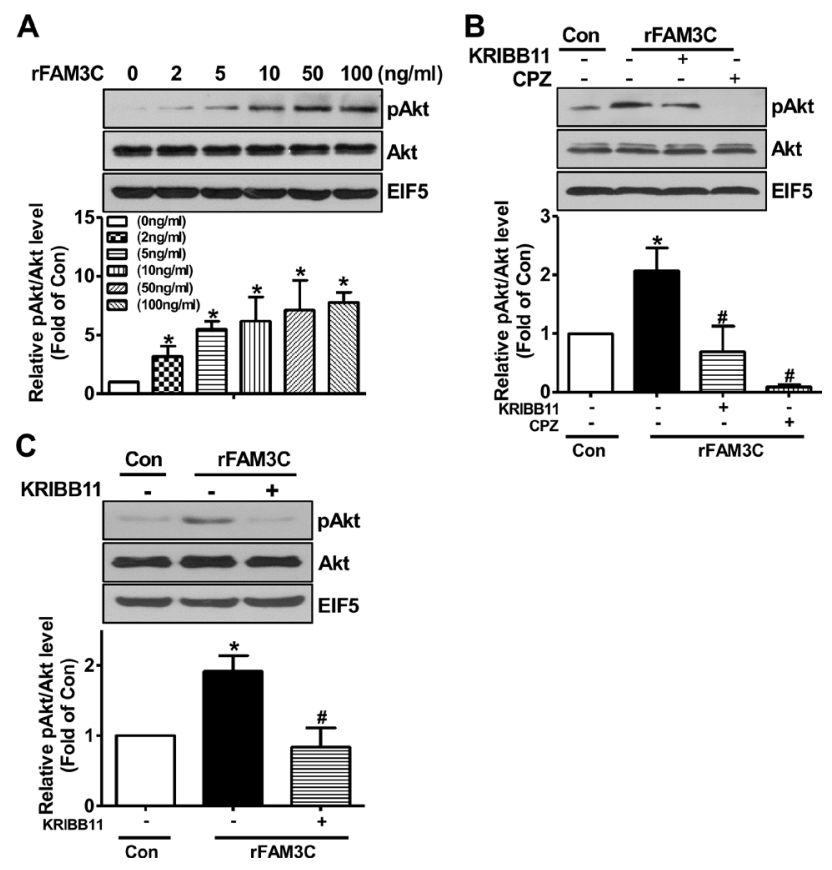

Figure 6: rFAM3C induced Akt phosphorylation in hepatocytes. (A) rFAM3C dose-dependently induced Akt activation in HepG2 cells. Cells were treated with various concentration of rFAM3C for 24 hours. (B) rFAM3C induced Akt activation in HSF1- and CaM-dependent manner in HepG2 cells. Cells were treated with 5ng/ml rFAM3C for 24 hours. (C) rFAM3C induced Akt activation in HSF1-dependent manner in primary mouse hepatocytes. Hepatocytes were treated with $5 \mathrm{ng} / \mathrm{ml} \mathrm{rFAM} 3 \mathrm{C}$ for 24 hours. The treatment of inhibitors was detailed in the experimental procedure. Con, control cells; rFAM3C, cells treated with rFAM3C. rFAM3C, recombinant human FAM3C protein. $\mathrm{N}=4,{ }^{*} \mathrm{P}<0.05$ versus control cells, ${ }^{\mathrm{P}} \mathrm{P}<0.05$ versus rFAM3C-treated cells without inhibitors. 

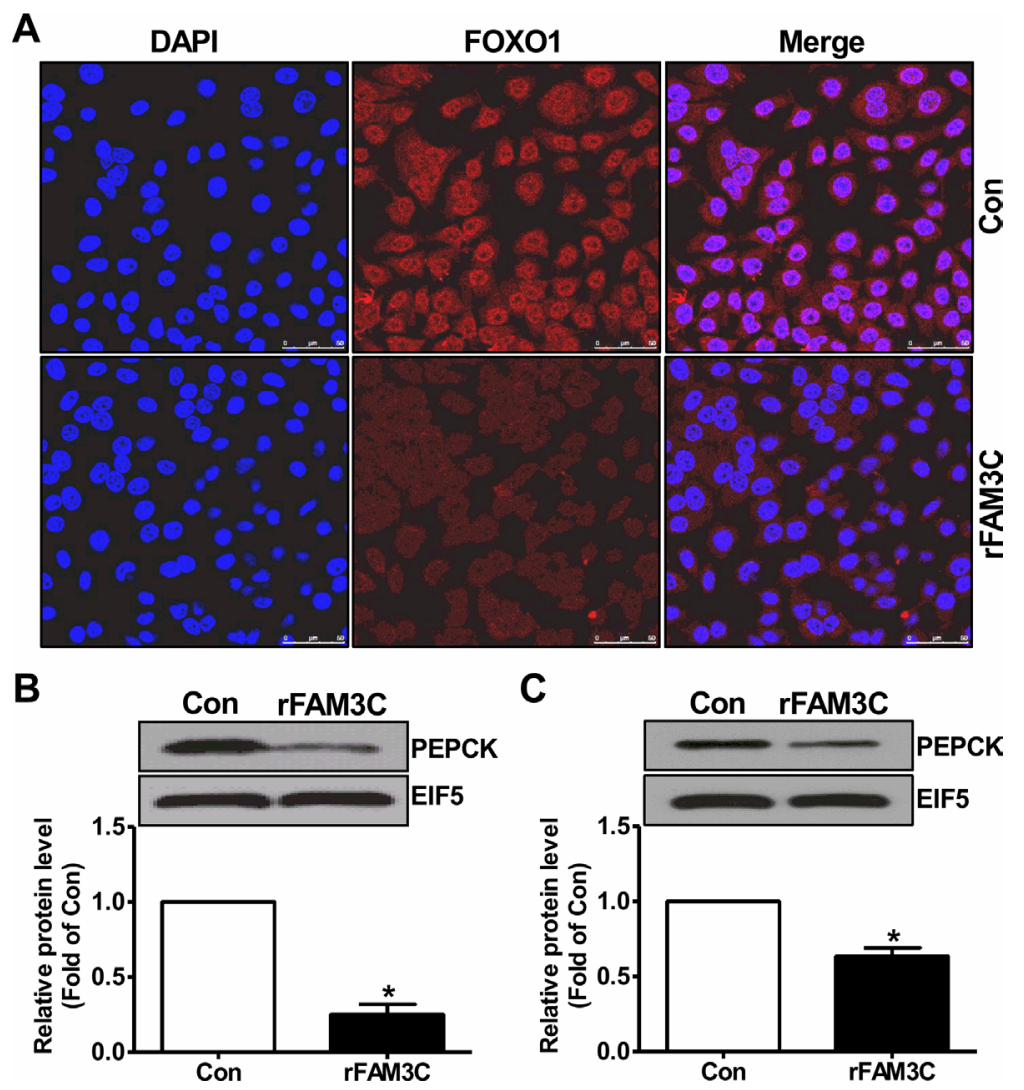

Figure 7: rFAM3C inactivated FOXO1 and repressed gluconeogenic gene expression in hepatocytes. Cells were treated with $5 \mathrm{ng} / \mathrm{ml} \mathrm{rFAM} 3 \mathrm{C}$ for 24 hours. (A) rFAM3C promoted the nuclear exclusion of FOXO1 in HepG2 cells. The images were the representatives of 3 experiments. (B) rFAM3C reduced PEPCK protein level in HepG2 cells. (C) rFAM3C reduced PEPCK protein level in primary mouse hepatocytes. Con, control cells; rFAM3C, cells treated with rFAM3C. $\mathrm{N}=4-5,{ }^{*} \mathrm{P}<0.05$ versus control cells.

A

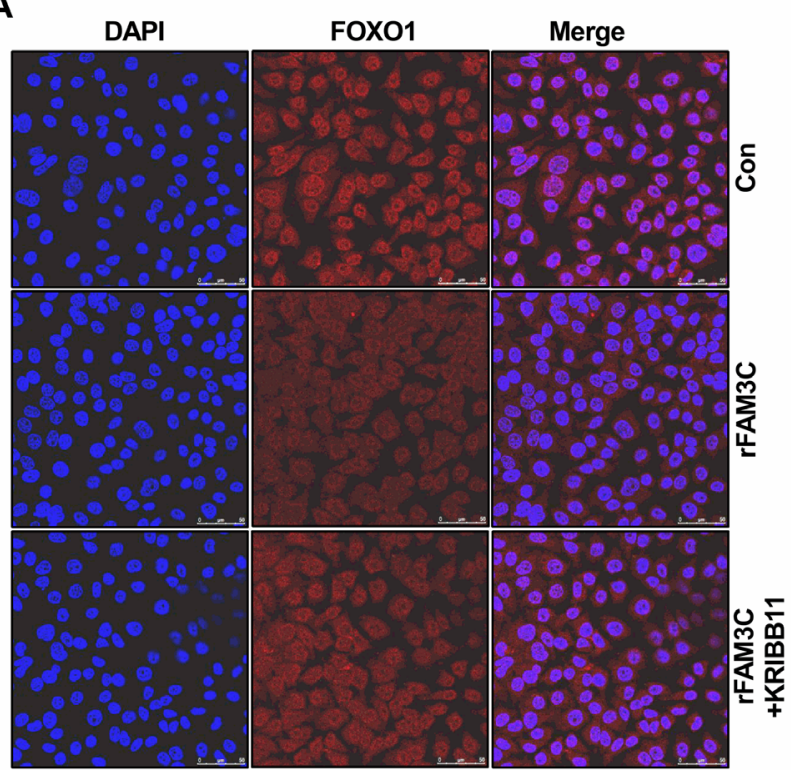

B

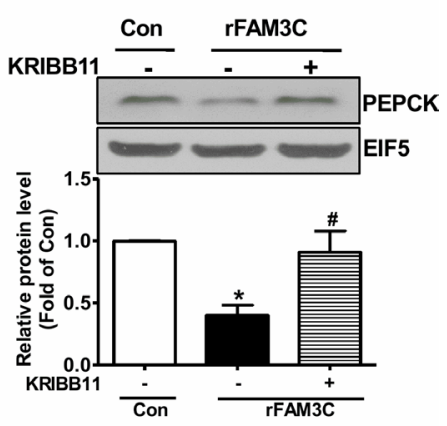

C

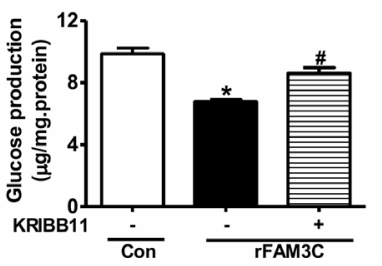

Figure 8: rFAM3C inactivated FOXO1 and repressed gluconeogenic gene expression in HSF1-dependent manner. Cells were treated with $5 \mathrm{ng} / \mathrm{ml} \mathrm{rFAM} 3 \mathrm{C}$ for 24 hours in the absence or presence of KRIBB11. (A) rFAM3C promoted the nuclear exclusion of FOXO1 in HSF1-dependent manner in HepG2 cells. The images were the representatives of 3 experiments. (B) rFAM3C repressed PEPCK protein expression in HSF1-dependent manner in HepG2 cells. (C) rFAM3C repressed gluconeogenesis in HSF1-dependent manner in HepG2 cells. Con, control cells; rFAM3C, cells treated with rFAM3C. N=4-5, ${ }^{*} \mathrm{P}<0.05$ versus control cells, ${ }^{\#} \mathrm{P}<0.05$ versus rFAM3C-treated cells without inhibitor. 
skeletal muscle and liver under diabetic condition. HSF 1 is the key transcriptor controlling the transcription of iHSP, and decreased HSF1 expression in main metabolic tissues is highly associated with insulin resistance and diabetes [34, 35]. In support, HSP72 expression was reduced in muscle under obese and insulin resistant status, and muscle-specific HSP72 overexpression protects against obesity-induced insulin resistance and glucose intolerance. Moreover, chaperone coinducer BGP-15 activates HSP72 to suppress hepatic glucose production, improve insulin resistance and hyperglycemia in obese diabetic mice [36]. Moreover, BGP-15 increases HSF1 binding activity and enhances the heat shock response through increased chromatin accessibility [37]. In the current and previous studies [19], we provided strong evidences that FAM3C function as a new hepatokine. FAM3C activates HSF1 to induce CALM1 expression and elevate CaM protein level, which finally promotes Akt phosphorylation and suppresses gluconeogenesis independent of insulin in hepatocytes. In contrast, FAM3C has little effect on CALM2 and CALM3 transcription [19]. CALM1, CALM2 and CALM3 encode one identical CaM protein. New FAM3C-HSF1-CaM-Akt metabolic pathway shed light on the understanding of the metabolic roles of HSF1. Interestingly, activation of Akt has also been reported to activate $\mathrm{HSF} 1$ [31, 32], suggesting that a cross-regulation exists between HSF1 and Akt. Overall, these findings together strongly suggested that HSF1 represents a novel therapeutical target for treating diabetes and other metabolic diseases.

In conclusion, FAM3C is a new hepatokine that activates HSF1-CaM-Akt pathway to ameliorate hyperglycemia of type 1 diabetic mice by suppressing hepatic gluconeogenesis in an insulin-independent manner (Figure 9). FAM3C-HSF1 axis is the potential target for the treatment of type 1 and type 2 diabetes.

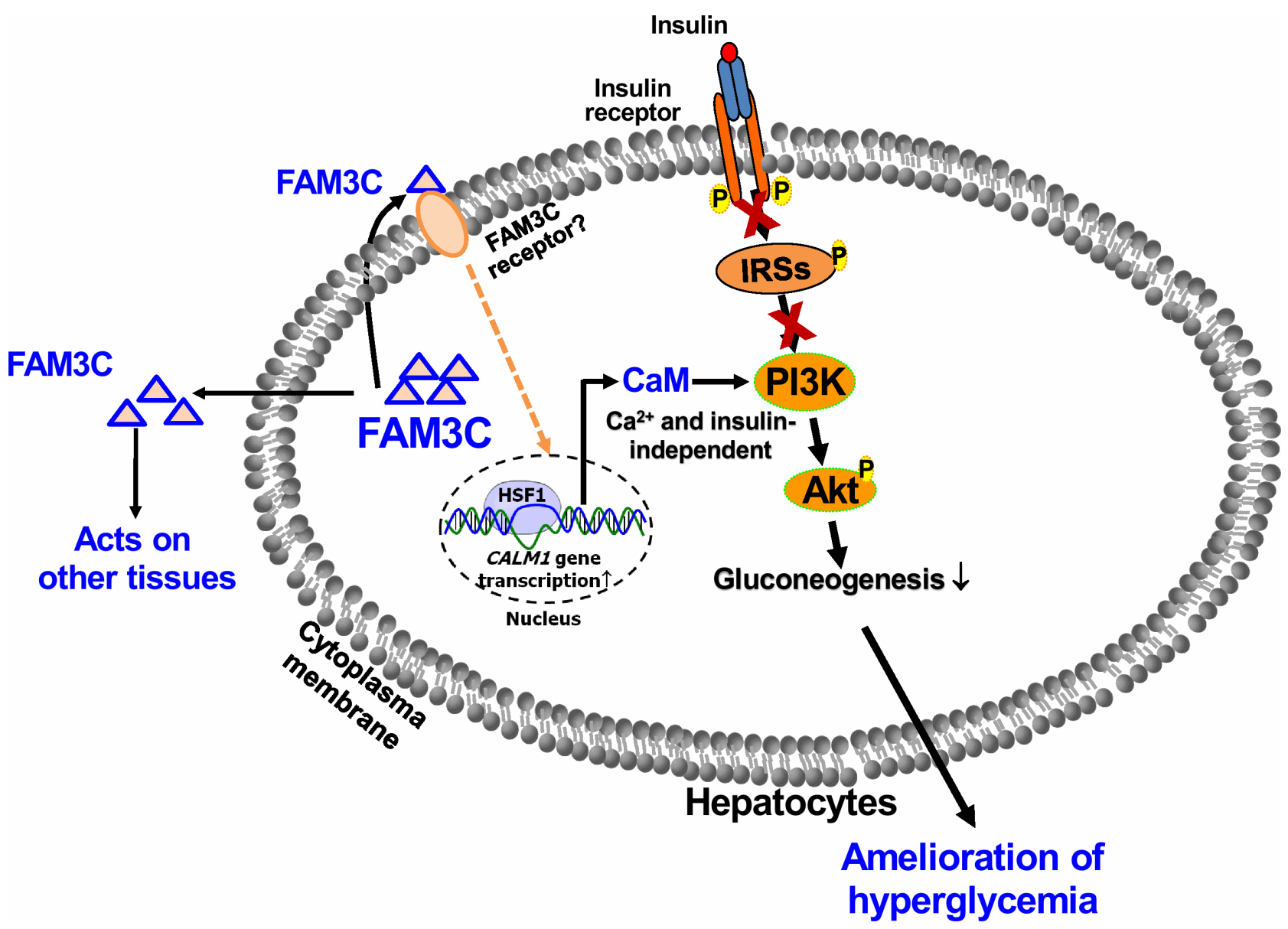

Figure 9: FAM3C functions as a hepatokine to suppress hepatic gluconeogenesis independent of insulin. $\mathrm{FAM} 3 \mathrm{C}$ is a new hepatokine that activates HSF1-CaM-PI3K pathway to induce Akt phosphorylation independent of insulin and calcium. Liver-secreted FAM3C may also act on other tissues beyond liver to exert its metabolic effects. Under severe insulin resistant and/or insulin deficient status, activating hepatic FAM3C-HSF1-Akt pathway represents a novel strategy for the treatment of diabetes. 


\section{MATERIALS AND METHODS}

\section{Construction of type 1 diabetic mouse models}

8-10 week old male C57BL/6 mice were used in the current study. Type 1 diabetes (T1D) was induced by intraperitoneal injection of streptozotocin (STZ) at the dose of $50 \mathrm{mg} / \mathrm{kg}$.bodyweight for 7 days. Mice with fasting blood glucose levels greater than $16.4 \mathrm{mmol} / \mathrm{L}$ were considered diabetic one month later. The control mice were treated with saline. Animal experiments were conducted in accordance with the Animal Management Rules of the Ministry of Health of the People's Republic of China and the guide for the Care and Use of the Laboratory Animals of the Peking University.

\section{Adenoviral overexpression of FAM3C in T1D mouse livers}

To overexpress FAM3C in T1D mouse livers, $1.0 \times 10^{9} \mathrm{pfu}$ of Ad-FAM3C or Ad-GFP were injected into mice via tail vein. Fasting blood glucose levels were monitored at the $4^{\text {th }}$ and $7^{\text {th }}$ day post viral injection. On the $8^{\text {th }}$ day, the mice were sacrificed on fed state for the experimental assays. The serum was collected for analyzing insulin levels. Liver and pancreas were taken for biochemical analyses.

\section{Hydrodynamics-based plasmid overexpression of HSF1 in T1D mouse livers}

Hydrodynamics-based transfection in animals by tail-vein administration of naked plasmid DNA was detailed previously $[16,19]$. In brief, STZ-treated diabetic mice were randomly divided into two groups based on fasting blood glucose levels. 50 $\mu$ g endotoxin-free pGFP or pHSF 1 was dissolved in sterile saline with the volume $10 \%$ of the bodyweight at room temperature, and then injected into tail vein in 7 seconds. Fasting blood glucose was monitored at 72 hours post plasmid injection. 24 hour later, mice were sacrificed for assays as above. The serum was collected for insulin detection.

\section{Conditioned medium and anti-FAM3C antibody treatment experiments}

HepG2 cells or mouse primary hepatocytes were infected with Ad-GFP or Ad-FAM3C for 24 hours, and then the cell culture medium was collected. The medium was centrifuged at $13,000 \mathrm{~g}$ at $4^{\circ} \mathrm{C}$ for 20 minutes, and the supernatant was collected as conditioned medium. Conditioned medium was added into cultured HepG2 cells or mouse hepatocytes to $1 / 3$ volume of the culture medium, and the cells were incubated for 24 hours before being lysed for Akt phosphorylation analyses. For antiFAM3C antibody treatment experiment, HepG2 cells were infected with Ad-FAM3C for 6 hours, and then incubated with anti-FAM3C antibodies (Abcam, ab72182) at the concentration of $2.5 \mu \mathrm{g} / \mathrm{ml}$ for 24 hours before pAkt or glucose production analyses. The procedure for gluconeogenesis assay in cultured hepatocytes was detailed in previous studies $[16,19]$.

\section{Treatment with recombinant human FAM3C protein}

Recombinant human FAM3C protein (rFAM3C) was purchased from Novoprotein (Cat\#:C343, secretory form, 22kD). Human HepG2 cells or primary mouse hepatocytes were treated with indicated concentration of rFAM3C for 24 hours before experimental analyses. For inhibiting HSF1, cells were treated with $\mathrm{FFAM} 3 \mathrm{C}$ in the absence or presence of KRIBB11 $(3 \mu \mathrm{M})$ for 24 hours. For inhibiting CaM, cells treated with rFAM3C for 24 hours were incubated with CPZ $(100 \mu \mathrm{M})$ for 1 hour before pAkt analyses.

\section{Primary mouse hepatocyte culture}

Livers were perfused in situ with $50 \mathrm{ml}$ of KRB buffer solution, followed by $30 \mathrm{ml}$ of liver digestion medium containing collagenase. The liver was excised, minced, and strained through a steel mesh. The dispersed hepatocytes were collected by centrifugation at $50 \mathrm{~g}$ for $3 \mathrm{~min}$ at $4^{\circ} \mathrm{C}$ for three times. Isolated hepatocytes were washed and plated in collagen-coated plates at $37^{\circ} \mathrm{C}, 5 \%$ $\mathrm{CO}_{2}$ for 6-8 $\mathrm{h}$ to allow for attachment, followed by the removal of unattached cells and treatment. Hepatocytes were treated with recombinant FAM3C protein for 24 hours before analyses.

\section{RNA extraction and real time-PCR assays}

Total RNA from HepG2 cells or tissues was prepared using TRIzol (Invitrogen) in accordance with the manufacturer's recommendations. $2 \mu \mathrm{g}$ total RNA was used in each cDNA synthesis reaction using cDNA Synthesis Kit (Thermo Scientific) with an oligo (dT) primer. Quantitative real-time PCR was performed using SYBR Green PCR Master Mix. The amplification was performed at $94^{\circ} \mathrm{C}$ for 2 minutes and subjected to 40 cycles of $94^{\circ} \mathrm{C}$ for 30 seconds, $59^{\circ} \mathrm{C}$ for 30 seconds, and $72^{\circ} \mathrm{C}$ for 30 seconds, followed by a final extension at $72^{\circ} \mathrm{C}$ for 6 minutes. The relative levels of the target gene mRNA transcripts to the $\beta$-actin were calculated by $2^{-\Delta \Delta C t}$ methodology as detailed previously $[15,16,21]$. All PCR primers were listed in previous study [19].

\section{Western blotting}

Cells or tissues were lysed in RIPA lysis buffer containing proteinase inhibitors, followed by centrifugation for 10 minutes at $4^{\circ} \mathrm{C} 12,000 \mathrm{rpm}$ to collect 
supernatants. Protein concentration was assessed with BCA protein assay kit. 40-100 $\mu$ g protein were separated by SDS-PAGE, followed by transferring to nitrocellulose membranes and blocking with 5\% fat-free milk in TBS for 1 hours. The membranes were incubated with primary antibodies overnight at $4^{\circ} \mathrm{C}$, followed by washing with TBS and incubating with a horseradish peroxidaseconjugated secondary antibody for 2 hours. Proteins were visualized with enhanced chemiluminescence technique. Akt phosphorylation was referred to phosphorylation at Ser473.

\section{Confocal analyses of FOXO1 nuclear exclusion}

Cells treated with recombinant FAM3C were permeabilized with $0.2 \%$ Triton X-100/0.5\% BSA, followed by washing with PBS. The coverslips were blocked in $1 \% \mathrm{BSA}$ for 30 minutes at $37^{\circ} \mathrm{C}$. The coverslips were incubated with anti-FOXO1 antibodies at $4^{\circ} \mathrm{C}$ overnight, and then washed with PBS, followed by detecting with goat anti-rabbit Alexa Fluor 594. After nuclear staining with DAPI, coverslips were mounted on glass slides using 50\% glycerol in PBS. Mounted coverslips were imaged and cells were visualized by fluorescence microscopy using Confocal Laser Scanning Microscope.

\section{Statistical analyses}

The results are presented as the mean \pm SEM. Statistical significance of differences between groups was analyzed by $t$-test. $\mathrm{P}$ values $<0.05$ were considered as statistically significant.

\section{Author contributions}

Z.C. and J.W. researched data and contributed to discussion. W.Y., J.C., Y.M., B.F. and Y.C. provided the technical assistance and animal model preparation. Z.C. and J.W. wrote the manuscript. B.G. and Q.C. contributed to discussion and reviewed/edited manuscript. Z.C., J.W. and J.Y. designed the study, and revised/edited manuscript.

\section{CONFLICTS OF INTEREST}

The authors declare that there is no conflicts of interest.

\section{FUNDING}

This study was supported by grants from National Key Research Program of China (2016YFC1304803 and 2017YFC0909600) the Natural Science Foundation of China (81670748/81471035/ 81670787) and Beijing Natural Science Foundation (7171006).

\section{REFERENCES}

1. Ogurtsova K, da Rocha Fernandes JD, Huang Y, Linnenkamp U, Guariguata L, Cho NH, Cavan D, Shaw JE, Makaroff LE. IDF Diabetes Atlas: global estimates for the prevalence of diabetes for 2015 and 2040. Diabetes Res Clin Pract. 2017; 128:40-50.

2. Rines AK, Sharabi K, Tavares CD, Puigserver P. Targeting hepatic glucose metabolism in the treatment of type 2 diabetes. Nat Rev Drug Discov. 2016; 15:786-804.

3. Roep BO, Tree TI. Immune modulation in humans: implications for type 1 diabetes mellitus. Nat Rev Endocrinol. 2014; 10:229-242.

4. Oh KJ, Han HS, Kim MJ, Koo SH. CREB and FoxO1: two transcription factors for the regulation of hepatic gluconeogenesis. BMB Rep. 2013; 46:567-574.

5. Mathieu C, Gillard P, Benhalima K. Insulin analogues in type 1 diabetes mellitus: getting better all the time. Nat Rev Endocrinol. 2017; 13:385-399.

6. Liu W, Yang XJ. The Effect of metformin on adolescents with type 1 diabetes: a systematic review and meta-analysis of randomized controlled trials. Int J Endocrinol. 2016; 2016:3854071.

7. Standl E. Metformin in type 1 diabetes. Lancet Diabetes Endocrinol. 2017.

8. Zhu Y, Xu G, Patel A, McLaughlin MM, Silverman C, Knecht K, Sweitzer S, Li X, McDonnell P, Mirabile R, Zimmerman D, Boyce R, Tierney LA, et al. Cloning, expression, and initial characterization of a novel cytokinelike gene family. Genomics. 2002; 80:144-150.

9. Li J, Chi Y, Wang C, Wu J, Yang H, Zhang D, Zhu Y, Wang N, Yang J, Guan Y. Pancreatic-derived factor promotes lipogenesis in the mouse liver: role of the Forkhead box 1 signaling pathway. Hepatology. 2011; 53:1906-1916.

10. Mo X, Yang C, Wang X, Burkhardt BR, Li Y, Xia H, Cao X. $\mathrm{F} 3 \mathrm{MB}(\mathrm{PANDER})$ decreases mice hepatic triglyceride and is associated with decreased DGAT1 expression. PLoS One. 2015; 10:e0117156.

11. Moak SL, Dougan GC, MarElia CB, Danse WA, Fernandez AM, Kuehl MN, Athanason MG, Burkhardt BR. Enhanced glucose tolerance in pancreatic-derived factor (PANDER) knockout C57BL/6 mice. Dis Model Mech. 2014; 7:1307-1315.

12. Yang J, Gao Z, Robert CE, Burkhardt BR, Gaweska H, Wagner A, Wu J, Greene SR, Young RA, Wolf BA. Structure-function studies of PANDER, an islet specific cytokine inducing cell death of insulin-secreting beta cells. Biochemistry. 2005; 44:11342-11352.

13. Yang J, Guan Y. Family with sequence similarity 3 gene family and nonalcoholic fatty liver disease. J Gastroenterol Hepatol. 2013; 28:105-111.

14. Yang J, Wang C, Li J, Burkhardt BR, Robert-Cooperman CE, Wilson C, Gao Z, Wolf BA. PANDER binds to the 
liver cell membrane and inhibits insulin signaling in HepG2 cells. FEBS Lett. 2009; 583:3009-3015.

15. Wang C, Chi Y, Li J, Miao Y, Li S, Su W, Jia S, Chen Z, Du S, Zhang X, Zhou Y, Wu W, Zhu M, et al. FAM3A activates PI3K p110alpha/Akt signaling to ameliorate hepatic gluconeogenesis and lipogenesis. Hepatology. 2014; 59:1779-1790.

16. Yang W, Wang J, Chen Z, Chen J, Meng Y, Chen L, Chang Y, Geng B, Sun L, Dou L, Li J, Guan Y, Cui Q, Yang J. NFE2 induces miR-423-5p to promote gluconeogenesis and hyperglycemia by repressing hepatic FAM3A-ATP-Akt pathway. Diabetes. 2017.

17. Zhou Y, Jia S, Wang C, Chen Z, Chi Y, Li J, Xu G, Guan Y, Yang J. FAM3A is a target gene of peroxisome proliferatoractivated receptor gamma. Biochim Biophys Acta. 2013; 1830:4160-4170.

18. Chen Z, Wang J, Yang W, Chen J, Meng Y, Geng B, Cui Q, Yang J. FAM3A mediates PPARgamma's protection in liver ischemia-reperfusion injury by activating Akt survival pathway and repressing inflammation and oxidative stress. Oncotarget. 2017; 8:49882-49896. https://doi.org/10.18632/ oncotarget. 17805.

19. Chen Z, Ding L, Yang W, Wang J, Chen L, Chang Y, Geng B, Cui Q, Guan Y, Yang J. Hepatic activation of the FAM3C-HSF1-CaM pathway attenuates hyperglycemia of obese diabetic mice. Diabetes. 2017; 66:1185-1197.

20. Hasegawa H, Liu L, Tooyama I, Murayama S, Nishimura M. The FAM3 superfamily member ILEI ameliorates Alzheimer's disease-like pathology by destabilizing the penultimate amyloid-beta precursor. Nat Commun. 2014; 5:3917.

21. Wang C, Chen Z, Li S, Zhang Y, Jia S, Li J, Chi Y, Miao Y, Guan Y, Yang J. Hepatic overexpression of ATP synthase beta subunit activates PI3K/Akt pathway to ameliorate hyperglycemia of diabetic mice. Diabetes. 2014; 63:947-959.

22. Boroughs LK, DeBerardinis RJ. Metabolic pathways promoting cancer cell survival and growth. Nat Cell Biol. 2015; 17:351-359.

23. Kraya AA, Piao S, Xu X, Zhang G, Herlyn M, Gimotty P, Levine B, Amaravadi RK, Speicher DW. Identification of secreted proteins that reflect autophagy dynamics within tumor cells. Autophagy. 2015; 11:60-74.

24. Waerner T, Alacakaptan M, Tamir I, Oberauer R, Gal A, Brabletz T, Schreiber M, Jechlinger M, Beug H. ILEI: a cytokine essential for EMT, tumor formation, and late events in metastasis in epithelial cells. Cancer Cell. 2006; 10:227-239.

25. Gronborg M, Kristiansen TZ, Iwahori A, Chang R, Reddy R, Sato N, Molina H, Jensen ON, Hruban RH, Goggins MG, Maitra A, Pandey A. Biomarker discovery from pancreatic cancer secretome using a differential proteomic approach. Mol Cell Proteomics. 2006; 5:157-171.

26. Pernicova I, Korbonits M. Metformin--mode of action and clinical implications for diabetes and cancer. Nat Rev Endocrinol. 2014; 10:143-156.

27. Yang J, Robert CE, Burkhardt BR, Young RA, Wu J, Gao $\mathrm{Z}$, Wolf BA. Mechanisms of glucose-induced secretion of pancreatic-derived factor (PANDER or FAM3B) in pancreatic beta-cells. Diabetes. 2005; 54:3217-3228.

28. Robert-Cooperman CE, Dougan GC, Moak SL, Athanason MG, Kuehl MN, Bell-Temin H, Stevens SM Jr, Burkhardt BR. PANDER transgenic mice display fasting hyperglycemia and hepatic insulin resistance. J Endocrinol. 2014; 220:219-231.

29. Cao X, Yang C, Lai F, Hong Z, Lin H, Liu J, Li Y. Elevated circulating level of a cytokine, pancreatic-derived factor, is associated with metabolic syndrome components in a Chinese population. J Diabetes Invest. 2016; 7:581-586.

30. Lai F, Chen Y, Lin H, Wang X, Zhu X, Li Y, Xiao H, Cao X. Pancreatic-derived factor impaired glucagon-like Peptide-1 production from GLUTag enterendorine L-cell line and intestines. Mol Cell Endocrinol. 2017; 452:110-119.

31. Hooper PL. Induction of heat shock proteins in the therapy of type 2 diabetes and metabolic syndrome. EBioMedicine. 2014; 1:14-15.

32. Hooper PL, Balogh G, Rivas E, Kavanagh K, Vigh L. The importance of the cellular stress response in the pathogenesis and treatment of type 2 diabetes. Cell Stress Chaperones. 2014; 19:447-464.

33. Saibil H. Chaperone machines for protein folding, unfolding and disaggregation. Nat Rev Mol Cell Biol. 2013; 14:630-642.

34. Kavanagh K, Flynn DM, Jenkins KA, Zhang L, Wagner JD. Restoring HSP70 deficiencies improves glucose tolerance in diabetic monkeys. Am J Physiol Endocrinol Metab. 2011; 300:E894-901.

35. Kavanagh K, Zhang L, Wagner JD. Tissue-specific regulation and expression of heat shock proteins in type 2 diabetic monkeys. Cell Stress Chaperones. 2009; 14:291-299.

36. Chung J, Nguyen AK, Henstridge DC, Holmes AG, Chan MH, Mesa JL, Lancaster GI, Southgate RJ, Bruce CR, Duffy SJ, Horvath I, Mestril R, Watt MJ, et al. HSP72 protects against obesity-induced insulin resistance. Proc Natl Acad Sci U S A. 2008; 105:1739-1744.

37. Budzynski MA, Crul T, Himanen SV, Toth N, Otvos F, Sistonen L, Vigh L. Chaperone co-inducer BGP-15 inhibits histone deacetylases and enhances the heat shock response through increased chromatin accessibility. Cell Stress Chaperones. 2017. 